\title{
LOCAL PROPERTIES OF SOFT SEMI*-OPEN SETS
}

\section{P. GNANACHANDRA}

Centre for Research and Post Graduate Studies in Mathematics, Ayya Nadar Janaki Ammal College (Autonomous), Sivakasi -626 124, Tamil Nadu, India

Corresponding author: pgchandra07@gmail.com

Received Mar. 31, 2020

AвSTRACT. Soft set theory was first introduced by Molodtsov [10] in the year 1999, as a general mathematical tool for dealing with problems that contain uncertainty. Soft set theory has a rich potential for applications in several directions. The notion of soft topological spaces was formulated by Shabir et al [5] and Cagman et al [2] separately in 2011. Robert and Pious Missier $[13,14]$ defined semi*-open and semi*-closed sets using generalized closure operator. Many researchers defined some basic notions on soft topology and studied many properties see [ [1], [5], [9], [11], [16]]. In this paper, we introduce and study soft semi*-connectedness and soft semi*-compactness using soft semi*-open sets.

2010 Mathematics Subject Classification. 54D10, 54D15.

Key words and phrases. soft semi*-closure; soft semi*-interior; soft generalized closure; soft generalized interior; soft semi*-connected; soft semi*-compact.

\section{Introduction}

Soft set theory was first introduced by Molodtsov [10] in 1999 as a general mathematical tool for dealing problems of incomplete information. He has shown several applications of this theory in solving many practical problems in economics, engineering, social sciences, medical sciences and so on. Soft set theory has a wider application and its progress is very rapid in different fields [see [1], [5], [9], [11] and [16]]. Muhmmad Shabir and Munazza Naz [15] introduced soft topological spaces and the notions of soft open sets, soft closed sets,

DOI: $10.28924 / A P J M / 7-17$ 
soft closure, soft interior points, soft neighborhood of a point and soft separation axioms. Kannan [7] introduced soft generalized closed sets in soft topological spaces. Juthika Mahanta and P.K.Das [6] introduced semi -open and semi -closed soft sets. Pious Missier and Robert [13,14] defined semi*-open and semi*-closed sets using generalized closure operator. In this work, we introduce and brood over soft semi*-compactness and soft semi*-connectedness using soft semi*-open sets.

\section{Preliminaries}

Let $U$ be an initial universe set and $E$ be a collection of all possible parameters with respect to $U$, where parameters are the characteristics or properties of objects in $U$. Let $P(U)$ denote the power set of $U$, and let $A \subseteq E$. Here are some definitions required in the sequel.

A subset $A$ of a space $(X, \tau)$ is said to be generalized closed [8] (briefly g-closed), if $c l(A) \subseteq U$ whenever $A \subseteq U$ and $U$ is open. The intersection of all g-closed sets containing $A$ is called the g-closure of $\mathrm{A}$ and denoted by $c l^{*}(A)[3]$. A subset $A$ of a space $(X, \tau)$ is said to be generalized open if its complement is generalized closed and union of all g-open sets contained in $A$ is called the g-interior of $A$ and is denoted by $i n t^{*}(A)$. A subset $S$ of a topological space $(X, \tau)$ is said to semi*-open [14] if $S \subseteq\left(c l^{*}(\operatorname{int}(S))\right.$. The complement of a semi*-open set is semi*-closed. It is well known that a subset $S$ is semi*-closed [13]if and only if $\operatorname{int}^{*}(\operatorname{cl}(S)) \subseteq S$ :

Definition 2.1. [10] $A$ soft set $F_{A}$ on the universe $U$ is defined by the set of ordered pairs $F_{A}=$ $\left\{\left(x, f_{A}(x)\right) \mid x \in E, f_{A}(x) \in P(U)\right\}$ where $E$ is a set of parameters, $A \subseteq E, P(U)$ is the power set of $U$ and $f_{A}: A \rightarrow P(U)$ such that $f_{A}(x)=\phi$ if $x \notin A$. Here $f_{A}$ is called an approximate function of the soft set $F_{A}$. The value of $f_{A}(x)$ may be arbitrary, some of them may be empty and some may have non-empty intersection.

Note that the set of all soft sets over $U$ is denoted by $S S(U)_{E}$.

For illustration, we consider an example which we present below:

Example 2.2. Suppose $U=$ set of all real numbers on the closed interval $[a, b]$.

$E=$ set of parameters. Each parameter is a word or a sentence.

$E=\{$ Compact, Closed, Connected, Open $\}$

In this case, to define a soft set means to point out closed set, connected set and so on. Let we consider below the same example in more detail. $U=\{x: a \leq x \leq b\}$ and $E=\left\{e_{1}, e_{2}, e_{3}, e_{4}\right\}$ where $e_{1}$ stands for the parameter 'compact',

$e_{2}$ stands for the parameter 'closed', 
$e_{3}$ stands for the parameter 'connected',

$e_{4}$ stands for the parameter 'open'.

Suppose that

$f\left(e_{1}\right)=\{A \subseteq[a, b]$ :Every open cover for $A$ in $[a, b]$ has finite subcover $\}$.

$f\left(e_{2}\right)=\{[\alpha, \beta] \subseteq[a, b]: \alpha, \beta \in R\}$

$f\left(e_{3}\right)=\{A \subseteq[a, b]:$ Seperation does not exists for $A$ in $[a, b]\}$

$f\left(e_{4}\right)=\{(\alpha, \beta) \subseteq[a, b]: \alpha, \beta \in R\}$

The soft set $F_{A}$ is a parametrized family of subsets of the set $U$. Consider the mapping $f$ in which $f\left(e_{1}\right)$ means set of all subsets of $U$ which are compact whose functional value is the set $\{A \subseteq[a, b]:$ Every open cover for $A$ in $[a, b]$ has finite subcover $\}$. Hence the soft set $F_{A}$ is the collection of approximations given below:

$F_{A}=\{($ compact,$\{A \subseteq[a, b]:$ Every open cover for $A$ in $[a, b]$ has finite subcover $\}),(C l o s e d,\{[\alpha, \beta] \subseteq$ $[a, b]: \alpha, \beta \in R\}),($ Connected, $\{A \subseteq[a, b]$ :separation does not exist for $A$ in $R\}),($ Open, $\{(\alpha, \beta) \subseteq$ $[a, b]: \alpha, \beta \in R\})\}$

Definition 2.3. [3] Let $\tilde{\tau}$ be a collection of soft sets over a universe $U$ with a fixed set E of parameters, then $\tilde{\tau} \subseteq S S(U)_{E}$ is called a soft topology on $U$ with a fixed set $E$ if

i. $\phi_{E}, U_{E}$ belong to $\tilde{\tau}$.

ii. The union of any number of soft sets in $\tilde{\tau}$ belongs to $\tilde{\tau}$.

iii. The intersection of any finite number of soft sets in $\tilde{\tau}$ belongs to $\tilde{\tau}$.

The pair $\left(U_{E}, \tilde{\tau}\right)$ is called a soft topological space. For an illustration let us consider the following example.

Definition 2.4. [7] Let $\left(U_{E}, \tilde{\tau}\right)$ be a soft topological space over $U$.

(i) A soft set $F_{B}$ is called a soft generalized closed in $U$ if $\operatorname{cl}\left(F_{B}\right) \tilde{\subseteq} F_{0}$ whenever $F_{B} \tilde{\subseteq} F_{0}$ and $F_{0}$ is soft open in $U$.

(ii) A soft set $F_{B}$ is called a soft generalized open in $U$ if its soft complement $F_{B}^{\tilde{C}}$ is soft generalized closed in $U$. Equivalently $G_{c} \tilde{\subseteq} \operatorname{int}\left(F_{B}\right)$ whenever $G_{c} \tilde{\subseteq} F_{B}$ and $G_{c}$ is soft closed in $U$.

Definition 2.5. [5] Let $\left(U_{E}, \tilde{\tau}\right)$ be a soft topological space over $U$.

(i) The soft generalized closure of a soft set $F_{B}$ is denoted by $c l^{*}\left(F_{B}\right)$ and it is defined as the soft intersection of all soft generalized closed sets contains $F_{B}$. 
(ii) The soft generalized interior of a soft set $F_{B}$ is denoted by int* $\left(F_{B}\right)$ and it is defined as the soft union of all soft generalized open sets contained in $F_{B}$.

Definition 2.6. [4] In a soft topological space $\left(U_{E}, \tilde{\tau}\right)$ a soft set

(i) $G_{C}$ is said to be semi*-open soft set if there exists an open soft set $H_{B}$ such that $H_{B} \tilde{\widetilde{\subseteq}} G_{C} \tilde{\widetilde{\subseteq}} c l^{*}\left(H_{B}\right)$.

(ii) $L_{A}$ is said to be semi*-closed soft set if there exists an closed soft set $K_{D}$ such that $\operatorname{int}^{*}\left(K_{D}\right) \tilde{\simeq} L_{A} \tilde{\simeq} K_{D}$.

Definition 2.7. [4] Let $G_{c}$ be a soft set in a soft topological space.

(i) The soft semi*-closure of $G_{c}$, is defined as $s s^{*} \operatorname{cl}\left(G_{c}\right)=\tilde{\cap}\left\{S_{F} / G_{c} \tilde{\subseteq} S_{F}\right.$ and $\left.S_{F} \in S^{*} C S S(U)_{E}\right\}$ is a soft set.

(ii) The soft semi*-interior of $G_{c}$, is defined as $s s^{*} \operatorname{int}\left(G_{c}\right)=\tilde{\cup}\left\{S_{F} / S_{F} \tilde{\subseteq} G_{c}\right.$ and $\left.S_{F} \in S^{*} O S S(U)_{E}\right\}$ is a soft set.

Thus $s^{*} c l\left(G_{c}\right)$ is the smallest semi*-closed soft set containing $G_{c}$ and ss*int $\left(G_{c}\right)$ is the largest semi*open soft set contained in $G_{c}$.

\section{Soft Semi*-Compactness}

The study on compactness for a soft topological space was initiated by Zorlutuna et al [16]. Generalization of open sets to semi*-open sets in soft topological spaces also demands generalization of compactness. This section is devoted to introduce semi*-compactness in soft topological spaces along with its characterization.

Definition 3.1. A cover of a soft set is said to be a semi*-open soft cover if every member of the cover is a semi*-open soft set.

Definition 3.2. A soft topological space $\left(U_{E}, \tau\right)$ is said to be semi*-compact if each semi*-open soft cover of $U_{E}$ has a finite sub cover.

Theorem 3.3. A soft topological space $\left(U_{E}, \tau\right)$ is semi*-compact if and only if each family of semi*closed soft sets in $U_{E}$ with the finite intersection property has a non empty intersection.

Proof. Assume that $\left(U_{E}, \tilde{\tau}\right)$ is a semi*-compact soft topological space. Let $\left\{\left(L_{A}\right)_{\lambda}: \lambda \in \Lambda\right\}$ be a collection of semi*-closed soft sets with the finite intersection property. If possible, assume that $\tilde{\bigcap}_{\lambda \in \Lambda}\left(L_{A}\right)_{\lambda}=\phi_{E}$. This implies $\tilde{U}\left(\left(L_{A}\right)_{\lambda}\right)^{c}=U_{E}$ So the collection $\left\{\left(\left(L_{A}\right)_{\lambda}\right)^{c}: \lambda \in \Lambda\right\}$ forms a soft semi*-open cover of $U_{E}$, which is soft semi*-compact. So, there exists a finite sub collection $\Delta$ of $\Lambda$ which 
also covers $U_{E}$. That is $\tilde{U}_{\lambda \in \Lambda}\left(\left(L_{A}\right)_{\lambda}\right)^{c}=U_{E}$. This implies $\tilde{U}_{\lambda \in \Lambda}\left(\left(L_{A}\right)\right)^{c}=\phi_{E}$. This is a contradiction to the finite intersection property. Hence $\bigcap_{\lambda \in \Lambda}\left(L_{A}\right)_{\lambda} \neq \phi_{E}$. Conversely, assume that each family of semi $i_{-}$ closed soft sets in $U_{E}$ with the finite intersection property has a non empty intersection. If possible let us assume $\left(U_{E}, \tau\right)$ is not semi*-compact. Then there exists a soft semi*-open cover $\left\{\left(G_{C}\right)_{\lambda}: \lambda \in \Lambda\right\}$ of $U_{E}$ such that for every finite sub collection $\Delta$ of $\Lambda$ we have $\tilde{U}_{\lambda \in \Delta}\left(G_{C}\right)_{\lambda} \neq U_{E}$. Implies $\bigcap_{\lambda \in \Delta}\left(\left(G_{C}\right)_{\lambda}\right)^{c} \neq \phi_{E}$. Hence $\left\{\left(\left(G_{C}\right)_{\lambda}\right)^{c}: \lambda \in \Lambda\right\}$ has a finite intersection property. So, by hypothesis $\bigcap_{\lambda \in \Lambda}\left(\left(G_{C}\right)_{\lambda}\right)^{c} \neq \phi_{E}$. Which implies $\bigcup_{\lambda \in \Lambda}\left(G_{C}\right)_{\lambda} \neq U_{E}$. This is a contradiction to our assumption. Therefore $\left(U_{E}, \tilde{\tau}\right)$ is a semi*-compact soft topological space.

Theorem 3.4. A soft topological space $\left(U_{E}, \tilde{\tau}\right)$ is semi*-compact if and only if for every family $\psi$ of soft sets with finite intersection property, $\bigcap_{G_{C} \in \psi} s^{*} c l\left(G_{C}\right) \neq \phi_{E}$.

Proof. Let $\left(U_{E}, \tilde{\tau}\right)$ be a semi*-compact soft topological space. If possible let us assume that $\bigcap_{G_{C} \in \psi} s s^{*} c l\left(G_{C}\right)=$ $\phi_{E}$ for some family $\psi$ of soft sets with the finite intersection property. So $\bigcup_{G_{C} \in \psi}\left(\operatorname{ss} s^{*} c l\left(G_{C}\right)\right)^{c}=U_{E}$. Hence $\Gamma=\left\{\left(s^{*} c l\left(G_{C}\right)\right)^{c}: G_{C} \in \psi\right\}$ forms an soft semi*-open cover for $U_{E}$. Then by semi*-compactness of $U_{E}$ there exists a finite subcover $\omega$ of $\psi$ such that $\tilde{U}_{G_{C} \in \omega}\left(s s^{*} c l\left(G_{C}\right)\right)^{c}=U_{E}$. We have $G_{C} \tilde{\subseteq} s s^{*} c l\left(G_{C}\right)$. Then $U_{E} \tilde{\subseteq} \tilde{U}_{G_{C} \in \omega}\left(G_{C}\right)^{c}$ and hence $U_{E}=\tilde{U_{C} \in \omega}\left(G_{C}\right)^{c}$. Therefore $\bigcap_{G_{C} \in \omega} G_{C}=\phi_{E}$. This is contradiction to the finite intersection property. Hence $\bigcap_{G_{C} \in \psi} s s^{*} c l\left(G_{C}\right) \neq \phi_{E}$.

Conversely, assume that $\bigcap_{G_{C} \in \psi} \operatorname{ss}{ }^{*} c l\left(G_{C}\right) \neq \phi_{E}$ for every family $\psi$ of soft sets with finite intersection property. Suppose assume that $\left(U_{E}, \tau\right)$ is not soft semi*-compact. Then there exists a family $\Gamma$ of semi*-open soft sets covering $U_{E}$ without a finite sub cover. So for every finite sub family $\omega$ of $\Gamma$ we

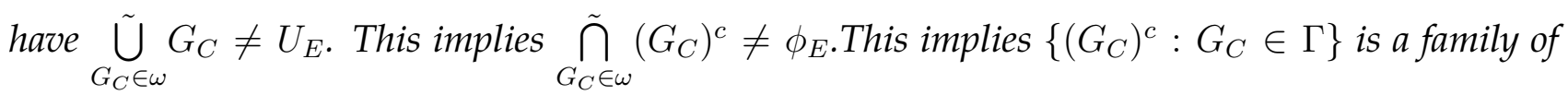
soft sets with finite intersection property. Now $\tilde{U}_{G_{C} \in \Gamma} G_{C}=U_{E}$. This implies $\tilde{\bigcap}_{G_{C} \in \Gamma}\left(G_{C}\right)^{c}=\phi_{E}$. Since $G_{C} \tilde{\subseteq} s s^{*} c l\left(G_{C}\right), \bigcap_{G_{C} \in \Gamma} s s^{*} c l\left(G_{C}\right)^{c} \tilde{\subseteq} \phi_{E}$. Hence $\bigcap_{G_{C} \in \Gamma} s s^{*} c l\left(G_{C}\right)^{c}=\phi_{E}$. This is a contradiction. Therefore $\left(U_{E}, \tilde{\tau}\right)$ is semi*-compact soft topological space.

Theorem 3.5. Semi*-continuous image of a soft semi*-compact space is soft compact.

Proof. Let $f: S S(U)_{E} \rightarrow S S(V)_{E^{\prime}}$ be a semi*-continuous function where $\left(U_{E}, \tilde{\tau}\right)$ is a semi*-compact soft topological space and $\left(V_{E^{\prime}}, \delta\right)$ is another soft topological space. Let $\left\{\left(G_{C}\right)_{\lambda}: \lambda \in \Lambda\right\}$ be a soft open cover of $V_{E^{\prime}}$. Since $f$ is semi*-continuous, $\left\{f^{-1}\left(G_{C}\right)_{\lambda}: \lambda \in \Lambda\right\}$ forms a soft semi*-open cover for $U_{E}$. This implies there exists a finite subset $\Delta$ of $\Lambda$ such that $\left\{f^{-1}\left(G_{C}\right)_{\lambda}: \lambda \in \Delta\right\}$ forms a soft semi*-open cover of $U_{E}$. Hence $\left\{\left(G_{C}\right)_{\lambda}: \lambda \in \Delta\right\}$ forms a finite soft sub cover of $V_{E^{\prime}}$. 
Theorem 3.6. Semi*-closed subspace of a semi*-compact soft topological space is soft semi*-compact. Proof. Let $V_{B}$ be a semi*-closed subspace of a semi*-compact soft topological space $\left(U_{E}, \tilde{\tau}\right)$ and $\left\{\left(G_{C}\right)_{\lambda}\right.$ : $\lambda \in \Lambda$ \} be a soft semi*-open cover for $V_{B}$. As $V_{B}$ is semi*-closed soft set $V_{B}^{c}$ is a semi*-open soft set. Hence $\Gamma=\left\{\left(G_{C}\right)_{\lambda}: \lambda \in \Lambda\right\} \tilde{U} V_{B}^{c}$ forms a semi*-open soft cover for $U_{E}$. As $U_{E}$ is soft semi*-compact $\Lambda$ has a finite sub family $\Delta$ such that $U_{E}=V_{B}^{c} \tilde{\bigcup}\left\{\left(G_{C}\right)_{\lambda}: \lambda \in \Delta\right\}$. Then $V_{B}=\left\{\left(G_{C}\right)_{\lambda}: \lambda \in \Delta\right\}$.

Theorem 3.7. Semi*-irresolute image of a semi*-compact soft topological space is semi*-compact.

Proof. Let $f: S S(U)_{E} \rightarrow S S(V)_{E^{\prime}}$ be a semi*-irresolute soft function where $\left(U_{E}, \tilde{\tau}\right)$ is a semi*-compact soft topological space and $\left(V_{E}^{\prime}, \delta\right)$ be a soft topological space. Let $\left\{\left(G_{C}\right)_{\lambda}: \lambda \in \Lambda\right\}$ be a soft semi*-open cover for $V_{E^{\prime}}$. As $f$ is a semi*-irresolute function $f^{-1}\left(G_{C}\right)_{\lambda}$ is a soft semi*-open set for each $\lambda \in \Lambda$. Hence $\left\{f^{-1}\left(G_{C}\right)_{\lambda}: \lambda \in \Lambda\right\}$ forms a semi*-open cover for $U_{E}$. Since $\left(U_{E}, \tau\right)$ is a semi*-compact, there exists a finite subfamily $\Delta$ of $\Lambda$ such that $\left\{f^{-1}\left(G_{C}\right)_{\lambda}: \lambda \in \Delta\right\}$ covers $\left(U_{E}, \tilde{\tau}\right)$. Hence $\left\{\left(G_{C}\right)_{\lambda}: \lambda \in \Delta\right\}$ forms a finite sub cover of $f\left(U_{E}\right)$. Hence $f\left(U_{E}\right)$ is soft semi*-compact.

\section{Soft Semi*-Connectedness}

Connectedness is one of the important notions of topology. In this section, we introduce semi*-connectedness in soft topological spaces using semi*-open soft sets and examine its basic properties.

Definition 4.1. [12] Two soft sets $L_{A}$ and $H_{B}$ are said to be disjoint if $L_{A}(a) \tilde{\cap} H_{B}(b)=\phi$ for all $a \in A, b \in B$.

Definition 4.2. A soft semi*-separation of soft topological $\left(U_{E}, \tau\right)$ is a pair $L_{A}, H_{B}$ of disjoint non null semi*-open sets whose union is $U_{E}$. If there does not exists a soft semi*-separation of $U_{E}$, then the soft topological space is said to be soft semi*-connected otherwise soft semi*-disconnected.

Example 4.3. Consider the soft topological space $\left(U_{E}, \tau\right)$, where $U=\left\{h_{1}, h_{2}\right\}, E=\left\{e_{1}, e_{2}\right\}$, and $\tau=\left\{\phi_{E}, U_{E},\left(e_{1},\left\{h_{1}\right\}\right),\left(e_{2},\left\{h_{1}, h_{2}\right\}\right),\left\{\left(e_{1},\left\{h_{1}\right\}\right)\right.\right.$,

$\left.\left(e_{2} \cdot\left\{h_{1}, h_{2}\right\}\right)\right\}$.The semi*-open soft sets are $\phi_{E}, U_{E},\left(e_{1},\left\{h_{1}\right\}\right),\left(e_{1},\left\{h_{1}, h_{2}\right\}\right)$, $\left\{\left(e_{1},\left\{h_{1}\right\}\right),\left(e_{2},\left\{h_{1}, h_{2}\right\}\right)\right\},\left(e_{2},\left\{h_{1}, h_{2}\right\}\right),\left\{\left(e_{1},\left\{h_{2}\right\}\right),\left(e_{2},\left\{h_{1}, h_{2}\right\}\right)\right\}$. None of the pairs is disjoint. So, there does not exist a soft semi*-separation of $U_{E}$, and hence is soft semi*-connected.

Theorem 4.4. If the soft sets $L_{A}$ and $G_{C}$ form a soft semi*-separation of $U_{E}$ and if $V_{B}$ is a soft semi*connected subspace of $U_{E}$ then $V_{B} \tilde{\subseteq} L_{A}$ or $V_{B} \tilde{\subseteq} G_{C}$.

Proof. Given $L_{A}$ and $G_{C}$ form a soft semi*-separation of $U_{E}$ Since $L_{A}$ and $G_{C}$ are disjoint semi*-open 
soft sets $L_{A} \tilde{\cap} V_{B}$ and $G_{C} \tilde{\cap} V_{B}$ are also semi*-open soft sets and their soft union gives $V_{B}$. That is they would constitute a soft semi*-separation of $V_{B}$. This is a contradiction. Hence one of $L_{A} \tilde{\cap} V_{B}$ and $G_{C} \tilde{\cap} V_{B}$ is empty. Therefore $V_{B}$ is entirely contained in one of them.

Theorem 4.5. Let $V_{B}$ be a soft semi*-connected subspace of $U_{E}$ and $K_{D}$ be a soft set in $U_{E}$ such that $V_{B} \tilde{\subseteq} K_{D} \tilde{\subseteq} c l\left(V_{B}\right)$ then $K_{D}$ is also soft semi*-connected.

Proof. Let the soft set $K_{D}$ satisfies the hypothesis. If possible, let $F_{A}$ and $G_{C}$ form a soft semi*-separation of $K_{D}$. Then by the theorem 5.4, $V_{B} \tilde{\subseteq} F_{A}$ or $V_{B} \tilde{\subseteq} G_{C}$. Let $V_{B} \tilde{\subseteq} F_{A}$. This implies $\operatorname{ss}{ }^{*} c l\left(V_{B}\right) \tilde{\subseteq} s s^{*} c l\left(F_{A}\right)$. Since $s s^{*} c l\left(F_{A}\right)$ and $G_{C}$ are disjoint, $V_{B}$ cannot intersects $G_{C}$. This is a contradiction. Hence $K_{D}$ is soft semi*-connected.

Theorem 4.6. A soft topological space $\left(U_{E}, \tilde{\tau}\right)$ is soft semi $i^{*}$-disconnected if and only if there exists a non null proper soft subset of $U_{E}$ which is both soft semi*-open and soft semi*-closed.

Proof. Let $U_{E}$ be soft semi*-disconnected. Then there exist non null soft subsets $K_{D}$ and $H_{C}$ Such that $s s^{*} c l\left(K_{D}\right) \tilde{\bigcap} H_{C}=\phi_{E}, K_{D} \tilde{\bigcap} s s^{*} c l\left(H_{C}\right)=\phi_{E}$ and $K_{D} \tilde{\cup} H_{C}=U_{E} \cdot \operatorname{Now} K_{D} \tilde{\subseteq} s s^{*} c l\left(K_{D}\right)$ and $s s^{*} c l\left(K_{D}\right) \tilde{\bigcap} H_{C}=\phi_{E}$.This implies $K_{D} \tilde{\bigcap} H_{C}=\phi_{E}$, that is $H_{C} \tilde{\subseteq}\left(K_{D}\right)^{c}$. Then $K_{D} \tilde{\bigcup} s s^{*} c l\left(H_{C}\right)=U_{E}$ and $K_{D} \tilde{\bigcap} s s^{*} c l\left(H_{C}\right)=\phi_{E}$ this implies $K_{D}=\left(s s^{*} c l\left(H_{C}\right)\right)^{c}$ similarly $H_{C}=\left(\operatorname{sic} c l\left(K_{D}\right)\right)^{c}$. Hence $K_{D}$ and $H_{C}$ are semi*-open soft sets being the complements of semi*-closed soft sets. Also $H_{C} \tilde{\subseteq}\left(K_{D}\right)^{c}$. This implies $K_{D}$ and $H_{C}$ are also semi*-closed soft sets. Conversely, let $K_{D}$ be a non null proper soft subset of $U_{E}$ which is both semi*-open and semi*-closed. Now let $H_{C} \tilde{\subseteq}\left(K_{D}\right)^{c}$ is non null proper subset of $U_{E}$ which is also both semi*-open and semi*-closed. This implies $U_{E}$ can be expressed as the soft union of two semi*-separated soft sets $K_{D}$ and $H_{C}$. Hence $U_{E}$ is semi*-disconnected.

Theorem 4.7. Semi*-irresolute image of a soft semi*-connected soft topological space is soft semi*connected.

Proof. Let Let $f: S S(U)_{E} \rightarrow S S(V)_{E^{\prime}}$ be a semi*-irresolute soft function where $\left(U_{E}, \tilde{\tau}\right)$ is a semi*connected soft topological space. Our aim is to prove is soft semi*-connected. Suppose assume that $f\left(U_{E}\right)$ soft semi*-disconnected. Let $K_{D}$ and $H_{C}$ be non null disjoint semi*-open soft sets whose union is $f\left(U_{E}\right)$. Since $f$ is semi*-irresolute soft function $f^{-1}\left(K_{D}\right)$ and $f^{-1}\left(H_{C}\right)$ are semi*-open soft sets. Also they form a soft semi*-separation for $U_{E}$. This is a contradiction to the fact that $U_{E}$ is soft semi*-connected. Hence $f\left(U_{E}\right)$ is soft semi*-connected.

Theorem 4.8. Semi*-continuous image of a soft semi*-connected soft topological space is soft connected. Proof. Let $f: S S(U)_{E} \rightarrow S S(V)_{E^{\prime}}$ be a semi*-continuous function where $\left(U_{E}, \tilde{\tau}\right)$ is a semi*-connected soft topological space and $\left(V_{E^{\prime}}, \delta\right)$ is a soft topological space. Our aim is to prove $f\left(U_{E}\right)$ is soft 
connected.Suppose assume that $f\left(U_{E}\right)$ is soft disconnected. Let $f\left(U_{E}\right)=K_{D} \tilde{U} H_{C}$ be a soft separation that is $K_{D}$ and $H_{C}$ are disjoint soft open sets whose union is $f\left(U_{E}\right)$. This implies $f^{-1}\left(K_{D}\right)$ and $f^{-1}\left(H_{C}\right)$ form a soft semi*-separation of $U_{E}$. This is a contradiction. Hence $f\left(U_{E}\right)$ is soft connected.

\section{Conclusion}

In this paper, we followed the work of Juthika mahanta et al. which is a step forward to further investigate the strong base of soft topological spaces. Further, we planned to introduce and investigate soft semi*-separation Axioms using soft semi*-open and soft semi*-closed sets.We assure that the belongings in this paper will help researchers move into the new direction and promote the future work in soft topological spaces.

\section{Acknowledgement}

The author thank Professor Saeid Jafari, College of Vestsjaelland South, Herrestraede 11, Slagelse, Denmark for his keen interest about this article and valuable suggestions.

\section{REFERENCES}

[1] A. Atgunoglu, H. Aygun, Some Notes on Soft Topological Spaces, Neural Comput. Appl. 21 (2012), 113-119.

[2] N. Cagman, S. Karatas, S. Enginoglu, Soft Topology, Comput. Math. Appl. 62 (2011), 351-358.

[3] W. Dunham, A New Closure Operator for Non-T1 Topologies, Kyungpook Math. J. 22 (1982), 55-60.

[4] P. Gnanachandra, M.L. Thivagar, Separation Axioms by virtue of Semi*-open sets, World Sci. News, 145 (2020), 74-84.

[5] S. Hussain, B. Ahmed,Some Properties of Soft Topological Spaces, Comput. Math. Appl. 62 (2011), 40484067.

[6] J. Mahanta, P.K. Das, On soft topological space via semi-open ansd semi-closed sets, Kyungpook Math. J. 54 (2014), 221-236.

[7] K. Kannan, Soft Generalized Closed Sets in Soft Topological Spaces, J. Theor. Appl. Technol. 37 (2012), $17-21$.

[8] N. Levine, Generalized closed sets in topology, Rend. Circ. Mat. Palermo, 19 (1970),89-96.

[9] W.K. Min, A note on Soft Topological Spaces, Comput. Math. Appl. 62 (2011), 3524-3528.

[10] D. Molodtsov, Soft Set Theory-First Results, Comput. Math. Appl. 37 (1999), 19-31.

[11] B.Pazar Varol.B and H Aygin,On Soft Hausdorff Spaces, Ann. Fuzzy Math. Inform. 5 (2013), 15-24.

[12] E. Peyghan, B. Samadi, A.Tayebi, On soft connectedness , arXiv:1202.1668V1 [math.GN], 2012.

[13] A. Robert, S.P. Missier, On Semi*-closed sets, Asian J. Eng. Math. 1 (4) (2012), 173-176. 
[14] S.P. Missier, A. Robert, On semi*-open sets, Int. J. Math. Soft Comput. 2 (2) (2012), 95-105.

[15] M. Shabir, M. Naz, On Soft Topological Spaces, Comput. Math. Appl. 61 (2011), 1786-1799.

[16] I. Zorlutuna, M.Akdag, W.K. Min, S. Atmaca, Remarks on Soft Topological Spaces, Ann. Fuzzy Math. Inform. 3 (2) (2012), 171-185. 